This item was submitted to Loughborough's Research Repository by the author.

Items in Figshare are protected by copyright, with all rights reserved, unless otherwise indicated.

\title{
Optimal weights in DEA models with weight restrictions
}

\section{PLEASE CITE THE PUBLISHED VERSION}

http://dx.doi.org/10.1016/j.ejor.2016.04.035

\section{PUBLISHER}

(c) Elsevier

\section{VERSION}

AM (Accepted Manuscript)

\section{PUBLISHER STATEMENT}

This work is made available according to the conditions of the Creative Commons Attribution-NonCommercialNoDerivatives 4.0 International (CC BY-NC-ND 4.0) licence. Full details of this licence are available at: https://creativecommons.org/licenses/by-nc-nd/4.0/

\section{LICENCE}

CC BY-NC-ND 4.0

\section{REPOSITORY RECORD}

Podinovski, Victor V.. 2019. "Optimal Weights in DEA Models with Weight Restrictions". figshare. https://hdl.handle.net/2134/21230. 


\title{
Optimal Weights in DEA Models with Weight Restrictions
}

\author{
Victor V. Podinovski ${ }^{\mathrm{a}}$ \\ ${ }^{a}$ School of Business and Economics, Loughborough University, Loughborough LE11 3TU, UK
}

\begin{abstract}
According to a conventional interpretation of a multiplier DEA model, its optimal weights show the decision making unit under the assessment, denoted $\mathrm{DMU}_{o}$, in the best light in comparison to all observed DMUs. For multiplier models with additional weight restrictions such an interpretation is known to be generally incorrect (specifically, if weight restrictions are linked or nonhomogeneous), and the meaning of optimal weights in such models has remained unclear. In this paper we prove that, for any weight restrictions, the optimal weights of the multiplier model show $\mathrm{DMU}_{o}$ in the best light in comparison to the entire technology expanded by the weight restrictions. This result is consistent with the fact that the dual envelopment DEA model benchmarks DMU against all DMUs in the technology, and not only against the observed DMUs. Our development overcomes previous concerns about the use of weight restrictions of certain types in DEA models and provides their rigorous and meaningful interpretation.
\end{abstract}

Keywords: data envelopment analysis, multiplier model, weight restrictions, production trade-offs 


\section{Introduction}

Data envelopment analysis (DEA) is a nonparametric approach to the assessment of efficiency and productivity of organizational units (Cooper et al. 2007, Thanassoulis et al. 2008). The latter are conventionally referred to as decision making units (DMUs). Standard DEA models are based on the assumption that the underlying production technology is characterized by either constant (CRS) or variable (VRS) returns to scale.

Both CRS and VRS models can be stated as two mutually dual linear programs referred to as the envelopment and multiplier models. The optimal value of these two programs is interpreted as the input or output radial efficiency of $\mathrm{DMU}_{o}$ under the assessment, depending on the orientation in which the models are solved (Charnes et al. 1978, Banker et al. 1984). In particular, in the envelopment model, $\mathrm{DMU}_{o}$ is benchmarked against the boundary of the CRS or VRS technology, and the radial efficiency of $\mathrm{DMU}_{o}$ is interpreted as the utmost proportional improvement factor to its input or output vector possible in the technology.

The multiplier models are stated in terms of variable input and output weights (multipliers). The CRS multiplier model can be shown to maximize the ratio of the total weighted output to the total weighted input (efficiency ratio) of $\mathrm{DMU}_{o}$, provided no such ratio across all observed DMUs can exceed the value of 1 . The VRS multiplier model has an additional dual variable interpretable in terms of returns to scale and scale elasticity (Banker et al. 1984, Podinovski et al. 2009, Podinovski and Førsund 2010, Sahoo and Tone 2015, Podinovski et al. 2016). As pointed by Charnes et al. (1978), the optimal input and output weights are the most favorable to $\mathrm{DMU}_{o}$ and show it in the best light in comparison to all observed DMUs.

\subsection{Weight restrictions}

Weight restrictions usually represent value judgements incorporated in the form of additional constraints on the input and output weights in the multiplier model. These constraints reduce the flexibility of weights and typically improve the discrimination of the DEA model (see, e.g., Allen et al. 1997, Thanassoulis et al. 2008, Cook and Zhu 2008, Joro and Korhonen 2015).

The use of weight restrictions generally changes the interpretation of efficiency in both the envelopment and multiplier models. From the technology perspective, the incorporation of weight restrictions results in the expansion of the model of technology (Charnes et al. 1989, Roll et al. 1991, Halme and Korhonen 2000). Podinovski (2004b) shows that this expansion is caused by the dual terms in the envelopment model generated by weight restrictions, and that $\mathrm{DMU}_{o}$ is projected on the boundary of the expanded technology. Therefore, $\mathrm{DMU}_{o}$ is benchmarked against all units in the technology (including those generated by the weight restrictions), and not only against the observed units.

The interpretation of efficiency in terms of the multiplier model with weight restrictions is somewhat less obvious and currently incomplete. This can be summarized as follows. If all weight restrictions are homogeneous and not linked (see Section 2 for a formal definition), the multiplier model correctly identifies the optimal weights (within the specified weight restrictions) that represent $\mathrm{DMU}_{o}$ in the best light in comparison to all observed DMUs (Podinovski 2001a).

However, a problem with the interpretation arises if at least one weight restriction is nonhomogeneous or is linked. In this case the optimal weights do not generally represent $\mathrm{DMU}_{o}$ in the best light in comparison to all observed DMUs. Consequently, the optimal value of the multiplier model with such weight restriction generally underestimates the relative efficiency of $\mathrm{DMU}_{o}$. Examples illustrating this point are given by Podinovski and Athanassopoulos (1998), Podinovski (1999, 2001a) and, recently, by Khalili et al. (2010). 


\subsection{Contribution}

In this paper we show that, for any weight restrictions, the optimal weights of the multiplier model show $\mathrm{DMU}_{o}$ in the best light in comparison to all DMUs in the expanded technology generated by the weight restrictions. This result is true if we search among all nonnegative input and output weights, or only among those that satisfy the weight restrictions.

Our results also overcome the discrepancy between the interpretation of the envelopment and multiplier models with weight restrictions. Indeed, as pointed above, the envelopment model benchmarks $\mathrm{DMU}_{o}$ against all DMUs in the technology expanded by the weight restrictions. However, the conventional interpretation of the multiplier model assumes that $\mathrm{DMU}_{o}$ should be benchmarked against the observed DMUs only. As noted, this conventional assumption does not lead to a meaningful interpretation of some types of weight restrictions. Our results show that the multiplier model does exactly the same as the envelopment modelit benchmarks $\mathrm{DMU}_{o}$ against all DMUs in the expanded technology, for all types of weight restrictions.

From a practical perspective, this new interpretation can be used to justify the incorporation of any types of weight restrictions in the multiplier model, and explain the meaning of the resulting optimal weights and efficiency scores. This includes absolute weight bounds and linked weight restrictions, whose meaning has so far remained unclear.

\section{Weight restrictions and production trade-offs}

To be specific, we derive our main results for the input-oriented models under the assumption of CRS. These results fully extend to the output-oriented models and also to the case of VRS, with obvious minor modifications as outlined in Section 5.

\subsection{Multiplier models with weight restrictions}

Consider the set of observed DMUs $\left(X_{j}, Y_{j}\right), j=1, \ldots, N$, where $X_{j} \in \mathbb{R}_{+}^{m} \backslash\{0\}$ and $Y_{j} \in \mathbb{R}_{+}^{s} \backslash\{0\}$ are, respectively, the vectors of inputs and outputs. The DMU $\mathrm{D}_{o}$ under the assessment is denoted $\left(X_{o}, Y_{o}\right)$.

Multiplier CRS models are stated in terms of variable vectors of input and output weights $v \in \mathbb{R}_{+}^{m}$ and $u \in \mathbb{R}_{+}^{s}$. Weight restrictions are additional constraints on vectors $v$ and $u$ incorporated in the multiplier model and stated in the general form as follows:

$$
Q_{t}^{\top} u-P_{t}^{\top} v \leq c_{t}, \quad t=1, \ldots, K
$$

In inequalities (1), components of vectors $Q_{t} \in \mathbb{R}^{s}$ and $P_{t} \in \mathbb{R}^{m}$, and constant scalars $c_{t}$ may be positive, negative or zero. The weight restriction $t$ is linked if both vectors $P_{t}$ and $Q_{t}$ are nonzero, and not linked otherwise. The weight restriction $t$ is homogeneous if $c_{t}=0$, and nonhomogeneous otherwise. ${ }^{1,2}$

\footnotetext{
${ }^{1}$ Following Charnes et al. (1989), unlinked homogeneous weight restrictions are often referred to as assurance regions of Type I. A special case of this type is virtual weight restrictions of Wong and Beasley (1990). Similarly, following Thompson et al. (1990), linked homogeneous weight restrictions are referred to as assurance regions of Type II. The most common example of non-homogeneous weight restrictions is absolute weight bounds (Dyson and Thanassoulis 1988).

${ }^{2}$ DEA literature suggests different methods for assessing weight restrictions of various types (see, e.g., reviews in Thanassoulis et al. 2009 and Jain et al. 2015). Our new results apply to any weight restrictions (1), regardless of the method used for their assessment.
} 
Remark 1. Using the normalizing equality of the multiplier models, any nonhomogeneous weight restriction can be replaced by a homogeneous one. For example, using equality (2.2) stated below, a nonhomogeneous weight restriction $t$ is replaced by the homogeneous (possibly linked) weight restriction which, after a simple rearrangement, takes on the form $Q_{t}^{\top} u-\left(P_{t}+c_{t} X_{o}\right)^{\top} v \leq 0 .^{3}$

Based on Remark 1 and therefore without loss of generality we assume that all weight restrictions (1) are homogeneous. The input-oriented CRS multiplier model with such weight restrictions is stated as follows:

$$
\begin{aligned}
\theta^{*}=\max & Y_{o}^{\top} u \\
\text { subject to } & X_{o}^{\top} v=1, \\
& Y_{j}^{\top} u-X_{j}^{\top} v \leq 0, \quad j=1, \ldots, N, \\
& Q_{t}^{\top} u-P_{t}^{\top} v \leq 0, \quad t=1, \ldots, K, \\
& u, v \geq 0 .
\end{aligned}
$$

\subsection{Envelopment models with production trade-offs}

To demonstrate that weight restrictions (2.4) result in the expansion of the standard CRS technology, consider the dual envelopment model to program (2):

$$
\begin{array}{ll}
\theta^{*}=\min & \theta \\
\text { subject to } & \sum_{j=1}^{N} \lambda_{j} X_{j}+\sum_{t=1}^{K} \pi_{t} P_{t}+S_{X}=\theta X_{o}, \\
& \sum_{j=1}^{N} \lambda_{j} Y_{j}+\sum_{t=1}^{K} \pi_{t} Q_{t}-S_{Y}=Y_{o}, \\
& \lambda, \pi, S_{X}, S_{Y} \geq 0, \theta \text { sign free. }
\end{array}
$$

The above model allows a straightforward interpretation. The DMU

$$
(\hat{X}, \hat{Y})=\left(\sum_{j=1}^{N} \lambda_{j} X_{j}, \sum_{j=1}^{N} \lambda_{j} Y_{j}\right)
$$

in equalities (3.2) and (3.3) is a unit in the standard CRS technology. DMU $(\hat{X}, \hat{Y})$ is further modified by the terms generated by weight restrictions (2.4):

$$
\left(P_{t}, Q_{t}\right), \quad t=1, \ldots, K
$$

These terms specify simultaneous changes to the inputs and outputs, and are implemented in proportions $\pi=\left(\pi_{1}, \ldots, \pi_{K}\right) \geq 0$. Finally, the slack vectors $S_{X}$ and $S_{Y}$ correspond to the assumption of free disposability of inputs and outputs.

Following Podinovski (2004b), the terms (4) are referred to as production trade-offs. By minimizing $\theta$, program (3) identifies the input radial projection of $\mathrm{DMU}_{o}$ on the boundary of the expanded CRS technology $\mathcal{T}_{\mathrm{CRS}-\mathrm{TO}}$ defined as follows.

\footnotetext{
${ }^{3}$ The described transformation obviously depends on the $\mathrm{DMU}_{o}$ under the assessment and also on the (input or output) orientation of the model (Podinovski 2004b, 2005).
} 
Definition 1 (Podinovski 2004b). Technology $\mathcal{T}_{\mathrm{CRS}-\mathrm{TO}}$ is the set of all pairs $(X, Y) \in \mathbb{R}_{+}^{m+s}$ for which there exist vectors $\lambda \in \mathbb{R}_{+}^{N}, \pi \in \mathbb{R}_{+}^{K}, S_{X} \in \mathbb{R}_{+}^{m}$ and $S_{Y} \in \mathbb{R}_{+}^{s}$, such that

$$
\begin{gathered}
X=\sum_{j=1}^{N} \lambda_{j} X_{j}+\sum_{t=1}^{K} \pi_{t} P_{t}+S_{X}, \\
Y=\sum_{j=1}^{N} \lambda_{j} Y_{j}+\sum_{t=1}^{K} \pi_{t} Q_{t}-S_{Y} .
\end{gathered}
$$

Remark 2. It is well known that, in some cases, the multiplier model (2) may be infeasible, which corresponds to an unbounded optimal value (i.e., equal to $-\infty$ ) of the dual envelopment model (3). Podinovski and Bouzdine-Chameeva $(2013,2015)$ prove that, in all such cases, the expanded technology $\mathcal{T}_{\mathrm{CRS}-\mathrm{TO}}$ allows free production, i.e., that there exists a $\operatorname{DMU}(\tilde{X}, \tilde{Y}) \in \mathcal{T}_{\mathrm{CRS}-\mathrm{TO}}$ such that $\tilde{X}=0$ and $\tilde{Y} \neq 0$. In this case weight restrictions (2.4) and trade-offs (4) are called inconsistent (with the data set formed by the observed DMUs). Podinovski and Bouzdine-Chameeva $(2013,2015)$ show that weight restrictions may be inconsistent even if program (2) is feasible and has a finite optimal value $\theta^{*}$ for all observed $\mathrm{DMU}_{o}$, and develop analytical and computational tests of consistency. If the weight restrictions are inconsistent, this usually points to an error in their assessment, and the weight restrictions should be reconsidered. In this paper we assume that weight restrictions (2.4) are consistent. In particular, this assumption implies that program (2) has a finite optimal value $\theta^{*}>0$, provided $X_{o} \neq 0$ and $Y_{o} \neq 0$.

\section{Motivational example}

The following example demonstrates that the weights $(u, v)$ that show $\mathrm{DMU}_{o}$ in the best light in comparison to all observed DMUs are generally different to the weights that show $\mathrm{DMU}_{o}$ in the best light in comparison to all DMUs in the expanded technology $\mathcal{T}_{\mathrm{CRS}-\mathrm{TO}}{ }^{4}$

Example 1. Table 1 shows two observed DMUs $A$ and $B$ in a model with one input and two outputs. (DMU $C$ is not observed and is introduced below.) Consider the multiplier CRS model for the assessment of the input radial efficiency of DMU $A$ in which we incorporate the additional linked weight restriction $4 u_{2}-v_{1} \leq 0:^{5}$

$$
\begin{aligned}
\theta^{*}=\max & 1 u_{1}+2 u_{2} \\
\text { subject to } & 1 v_{1}=1 \\
& 1 u_{1}+2 u_{2}-1 v_{1} \leq 0 \\
& 2 u_{1}+1 u_{2}-1 v_{1} \leq 0 \\
& 4 u_{2}-1 v_{1} \leq 0 \\
& u_{1}, u_{2}, v_{1} \geq 0
\end{aligned}
$$

Figure 1 illustrates the feasible region of program (6) in dimensions $u_{1}$ and $u_{2}$. (By (6.2), $v_{1}=1$ in any feasible solution and is not shown.) As seen from the graph, the unique optimal solution to program (6) is $u_{1}^{*}=0.375, u_{2}^{*}=0.25$, together with $v_{1}^{*}=1$ (shown as point $U)$. The corresponding optimal value of program (6) is $\theta^{*}=0.875$.

\footnotetext{
${ }^{4}$ A larger example with a similar observation is considered by Podinovski and Athanassopoulos (1998).

${ }^{5}$ As noted in Remark 1, equality (6.2) implies that in program (6) the weight restriction (6.5) is equivalent to the weight bound $u_{2} \leq 0.25$.
} 
Table 1: DMUs in Example 1.

\begin{tabular}{llll}
\hline DMU & Input & Output 1 & Output 2 \\
\hline$A$ & 1 & 1 & 2 \\
$B$ & 1 & 2 & 1 \\
$C$ & 1 & 0 & 4 \\
\hline
\end{tabular}

Benchmarking against the observed DMUs only. It is straightforward to show that the optimal weights $\left(u_{1}^{*}, u_{2}^{*}, v_{1}^{*}\right)$ do not represent DMU $A$ in the best light in comparison to all observed DMUs, in the sense discussed in Charnes et al. (1978) which is now considered the standard interpretation. Indeed, the efficiency ratios of observed DMUs $A$ and $B$ are defined as follows:

$$
E_{A}(u, v)=\frac{1 u_{1}+2 u_{2}}{1 v_{1}}, \quad E_{B}(u, v)=\frac{2 u_{1}+1 u_{2}}{1 v_{1}} .
$$

Substituting the above optimal weights into (7), we obtain $E_{A}\left(u^{*}, v^{*}\right)=0.875$ and $E_{B}\left(u^{*}, v^{*}\right)=1$. Therefore, judging by the optimal solution to program (6), DMU $A$ should be regarded as inefficient.

However, consider another feasible solution to program (6): $\hat{u}_{1}=0$ and $\hat{u}_{2}=0.25$, together with $\hat{v}_{1}=1$ (point $V$ in Figure 1). Substituting these weights into (7), we obtain $E_{A}(\hat{u}, \hat{v})=0.5$ and $E_{B}(\hat{u}, \hat{v})=0.25$. This implies that, for the output weights represented by point $V$, DMU $A$ has the maximum efficiency ratio among all observed DMUs and should therefore be regarded as efficient.

It is now clear that the optimal value 0.875 of the multiplier model (6) does not correctly represent the highest efficiency ratio that DMU $A$ can achieve in comparison to all observed DMUs. In this sense, the optimal weights $\left(u_{1}^{*}, u_{2}^{*}, v_{1}^{*}\right)$ are not the most favorable for DMU $A$.

The above problem was first identified by Podinovski and Athanassopoulos (1998) and Podinovski $(1999,2001)$ who developed a methodology that correctly benchmarked any $\mathrm{DMU}_{o}$ against the observed DMUs. ${ }^{6}$

In this paper we take a different route and show that the above optimal weights $\left(u_{1}^{*}, u_{2}^{*}, v_{1}^{*}\right)$ are, in fact, meaningful, but in a different sense. Namely, instead of showing DMU $A$ in the best light in comparison to the observed DMUs only, they benchmark this DMU against the entire technology $\mathcal{T}_{\mathrm{CRS}-\mathrm{TO}}$. This is illustrated below.

Benchmarking against the entire technology. As shown in Section 2, the weight restriction (6.5) generates the trade-off $P=(1), Q=(0,4)^{\top}$. By Definition 1, technology $\mathcal{T}_{\mathrm{CRS}-\mathrm{TO}}$ consists of all nonnegative DMUs $\left(x_{1}, y_{1}, y_{2}\right)$ that can be stated in the following form:

$$
\begin{aligned}
& x_{1}=1 \lambda_{A}+1 \lambda_{B}+1 \pi_{1}+S_{x_{1}}, \\
& y_{1}=1 \lambda_{A}+2 \lambda_{B}-S_{y_{1}}, \\
& y_{2}=2 \lambda_{A}+1 \lambda_{B}+4 \pi_{1}-S_{y_{2}},
\end{aligned}
$$

where $\lambda_{A}, \lambda_{B}, \pi_{1}, S_{x_{1}}, S_{y_{1}}, S_{y_{2}} \geq 0$.

\footnotetext{
${ }^{6}$ Podinovski (2001b, 2004a) explores this issue further and identifies particular types of weight restrictions, for which the optimal weights in the multiplier model are not the most favorable for $\mathrm{DMU}_{o}$. A summary of some of these results and their practical implications are discussed in Dyson et al. (2001).
} 


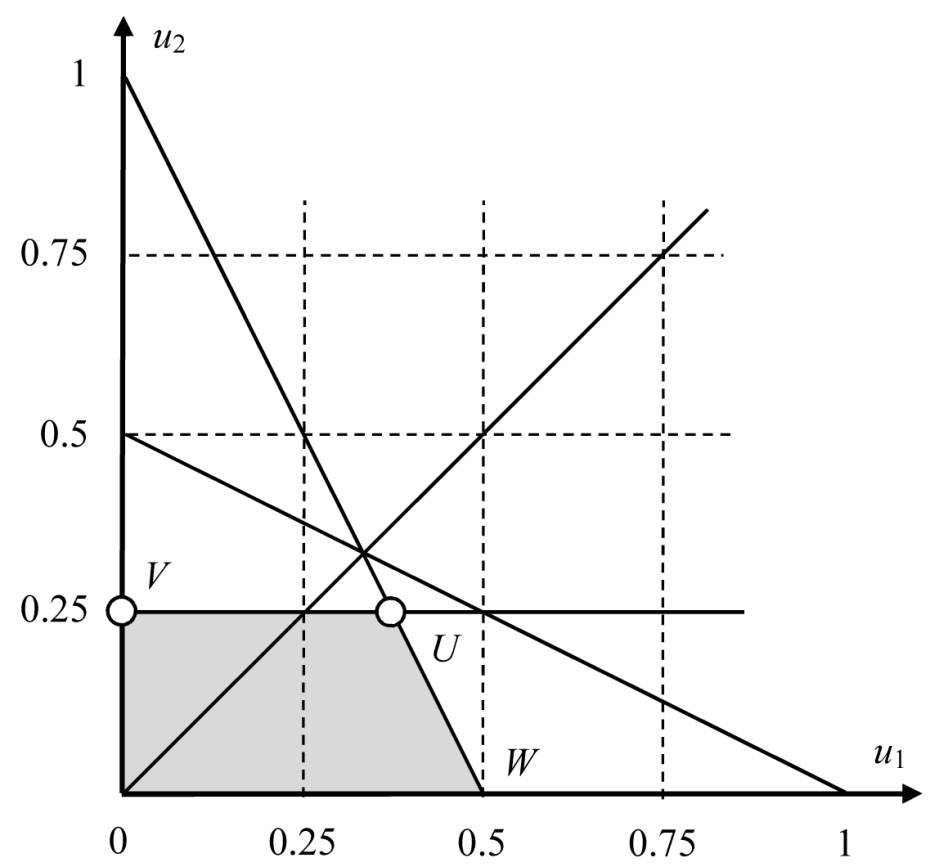

Figure 1: A graphical solution of Example 1. The output weights at point $U=(0.375,0.25)$ are optimal (together with $v_{1}=1$ ) in program (6). By Theorem 2, these weights represent DMU $A$ in the best light in comparison to all DMUs in technology $\mathcal{T}_{\mathrm{CRS}-\mathrm{TO}}$. The weights at point $V=(0,0.25)$ are not optimal in program (6) and represent DMU $A$ in the best light in comparison to the set of observed DMUs only.

Table 2: Efficiency ratios for different multiplier weights in Example 1.

\begin{tabular}{lll}
\hline DMU & Point $U:$ & Point $V:$ \\
& $u_{1}^{*}=0.375, u_{2}^{*}=0.25, v_{1}^{*}=1$ & $\hat{u}_{1}=0, \hat{u}_{2}=0.25, \hat{v}_{1}=1$ \\
\hline$A$ & 0.875 & 0.5 \\
$B$ & 1 & 0.25 \\
$C$ & 1 & 1 \\
\hline
\end{tabular}

Consider DMU $C$ in Table 1. It satisfies (8) with $\pi_{1}=1$ and $\lambda_{A}=\lambda_{B}=S_{x_{1}}=S_{y_{1}}=$ $S_{y_{2}}=0$. Therefore, $C \in \mathcal{T}_{\mathrm{CRS}-\mathrm{TO}}$. The efficiency ratio of DMU $C$ at both points $U$ and $V$ is equal to 1.

Table 2 shows the efficiency ratios of the three DMUs, $A, B$ and $C$, at the two sets of weights. Observe that, although the weights $\left(\hat{u}_{1}, \hat{u}_{2}, \hat{v}_{1}\right)$ represent DMU $A$ in the best light in comparison to DMU $B$ (and, therefore, in comparison to all observed DMUs in our example), these weights are not the best if $A$ is compared to $C$. Indeed, at point $U$ the efficiency ratio of $A$ is equal to 0.875 of the efficiency ratio of $C$, while at point $V$ it is only 0.5 of the latter.

The above example motivates our main development below. Namely, Theorem 2 formally establishes that the optimal weights $\left(u_{1}^{*}, u_{2}^{*}, v_{1}^{*}\right)$ of program (6) represent DMU $A$ in the best light in comparison to all DMUs in the expanded technology (8). Furthermore, the optimal value 0.875 is the highest efficiency ratio that DMU $A$ can achieve if benchmarked against all DMUs in this technology, and not only against the set of observed DMUs. 


\section{The main results}

In this section we show that any optimal weights $\left(u^{*}, v^{*}\right)$ to the multiplier model (2) show $\mathrm{DMU}_{o}$ in the best light in comparison to all units in technology $\mathcal{T}_{\mathrm{CRS}-\mathrm{TO}}$. As demonstrated by Example 1, this interpretation is generally not true if $\mathrm{DMU}_{o}$ is benchmarked against the set of observed DMUs only.

The above interpretation is clarified and supported by the results that we prove for three different variants of the multiplier model. This includes the multiplier model with linear constraints, its linear fractional analogue in which the maximum efficiency ratio is bounded above by 1 , and in the maximin model in which no normalization is required.

In the interests of generality, we assume that $\mathrm{DMU}_{o}$ is any unit in technology $\mathcal{T}_{\mathrm{CRS}-\mathrm{TO}}$ such that $X_{o} \in \mathbb{R}_{+}^{m} \backslash\{0\}$ and $Y_{o} \in \mathbb{R}_{+}^{s} \backslash\{0\}$. In particular, DMU may be any of the observed units.

\subsection{The linear multiplier model}

Consider the following program with the infinite number of linear constraints - note, this program is different from the standard multiplier model (2):

$$
\begin{aligned}
\theta_{1}^{*}=\max & Y_{o}^{\top} u \\
\text { subject to } & X_{o}^{\top} v=1, \\
& Y^{\top} u-X^{\top} v \leq 0, \quad \forall(X, Y) \in \mathcal{T}_{\mathrm{CRS}-\mathrm{TO}}, \\
& u, v \geq 0 .
\end{aligned}
$$

Denote $\Omega$ and $\Omega^{*}$ the sets of feasible and, respectively, optimal solutions to program (2). Similarly, let $\Omega_{1}$ and $\Omega_{1}^{*}$ be the sets of feasible and, respectively, optimal solutions to program (9).

Theorem 1. If solution $(u, v)$ is feasible in program (2), then $(u, v)$ is feasible in (9). If $\left(u^{*}, v^{*}\right)$ is optimal in $(2)$, then $\left(u^{*}, v^{*}\right)$ is optimal in (9). Therefore, the maximum in program (9) is attained, $\Omega \subseteq \Omega_{1}, \Omega^{*} \subseteq \Omega_{1}^{*}$ and $\theta^{*}=\theta_{1}^{*}$.

Theorem 1 and the other results are proved in Appendix A.

Proposition 1. For the projected $\operatorname{DMU}(X, Y)=\left(\theta^{*} X_{o}, Y_{o}\right) \in \mathcal{T}_{\mathrm{CRS}-\mathrm{TO}}$ and for any optimal weights $\left(u^{*}, v^{*}\right)$ to program (9), the inequality (9.3) is satisfied as equality, i.e., $Y_{o}^{\top} u^{*}-$ $\theta^{*} X_{o}^{\top} v^{*}=0$

Proposition 1 implies that the equation $Y^{\top} u^{*}-X^{\top} v^{*}=0$ defines a supporting hyperplane to technology $\mathcal{T}_{\mathrm{CRS}-\mathrm{TO}}$ at the projection $\left(\theta^{*} X_{o}, Y_{o}\right)$, and that $\left(-v^{*}, u^{*}\right)$ is its normal vector, where $\left(u^{*}, v^{*}\right)$ are any optimal weights in program (9) or, by Theorem 1 , in program (2).

Example 2 given in Appendix B shows that the feasible region of program (9) may be larger than the feasible region of program (2), i.e., generally, $\Omega \neq \Omega_{1}$. Similarly, in the general case, $\Omega^{*} \neq \Omega_{1}^{*}$. However, in an important special case we have the following result.

Proposition 2. Let all components of vectors $X_{o}$ and $Y_{o}$ be strictly positive. Then $\Omega^{*}=\Omega_{1}^{*}$.

The implications of Theorem 1 for the interpretation of optimal weights $\left(u^{*}, v^{*}\right)$ become clearer if program (9) is restated in a linear fractional form, as shown below. 


\subsection{The linear fractional model}

Similar to the standard CRS model, program (9) can be restated in the linear fractional form: ${ }^{7}$

$$
\begin{aligned}
\theta_{2}^{*}=\max & Y_{o}^{\top} u / X_{o}^{\top} v \\
\text { subject to } & Y^{\top} u / X^{\top} v \leq 1, \quad \forall(X, Y) \in \mathcal{T}_{\mathrm{CRS}-\mathrm{TO}} \\
& u, v \geq 0
\end{aligned}
$$

Theorem 2. If solution $\left(u^{*}, v^{*}\right)$ is optimal in program $(2)$, then $\left(u^{*}, v^{*}\right)$ is optimal in program (10). Therefore, the maximum in program (10) is attained and $\theta^{*}=\theta_{2}^{*}$.

The following statement is a useful analogue of Proposition 1.

Proposition 3. For the projected $\operatorname{DMU}(X, Y)=\left(\theta^{*} X_{o}, Y_{o}\right) \in \mathcal{T}_{\mathrm{CRS}-\mathrm{TO}}$ and for any optimal weights $\left(u^{*}, v^{*}\right)$ to program (10), the inequality (10.2) is satisfied as equality, i.e., $\left(Y_{o}^{\top} u^{*}\right) /\left(\theta^{*} X_{o}^{\top} v^{*}\right)=1$.

By Theorem 2, any optimal weights $\left(u^{*}, v^{*}\right)$ to the multiplier model with weight restrictions (2) maximize the efficiency ratio $Y_{o}^{\top} u / X_{o}^{\top} v$ of $\mathrm{DMU}_{o}$ with respect to the efficiency ratios of all DMUs in the expanded technology $\mathcal{T}_{\mathrm{CRS}-\mathrm{TO}}$, provided no such ratio exceeds the value of 1 . Using Proposition 3, we can change the last condition to the requirement that the maximum of efficiency ratios across all DMUs is equal to 1. Furthermore, program (10) does not explicitly incorporate weight restrictions (2.4), although these are implicitly accounted for in the definition of technology $\mathcal{T}_{\text {CRS-TO and, therefore, in constraints }}(10.2)$.

The above means that any optimal weights $\left(u^{*}, v^{*}\right)$ to the multiplier model $(2)$ represent $\mathrm{DMU}_{o}$ in the best light in comparison to all DMUs in the technology $\mathcal{T}_{\mathrm{CRS}-\mathrm{TO}}$. Furthermore, such weights are the most favorable for $\mathrm{DMU}_{o}$ among all nonnegative weights, and not only among those that satisfy weight restrictions (2.4).

Note that, as shown in Example 1, this interpretation does not imply that the optimal weights $\left(u^{*}, v^{*}\right)$ maximize the efficiency ratio of $\mathrm{DMU}_{o}$ with respect to observed DMUs only.

\subsection{The maximin model}

The constraints of program (10) normalize the maximum efficiency ratio of all DMUs by 1. As proved below, these conditions can be removed and program (10) restated in the following form analogous to the maximin program in Podinovski and Athanassopoulos (1998) and Podinovski (2001a): ${ }^{8}$

\footnotetext{
${ }^{7}$ Because program (10) may involve division by zero, we need to clarify the definition of its feasible region. A seemingly simple approach would require that we have $X^{\top} v>0$, for all DMUs $(X, Y) \in \mathcal{T}_{\mathrm{CRS}-\mathrm{TO}}$, and that all inequalities $(10.2)$ be true. However, this definition does not work well for several reasons. In particular, no weights satisfy it at the zero DMU $(0,0) \in \mathcal{T}_{\mathrm{CRS}-\mathrm{TO}}$, the maximum of program (10) becomes generally unattained, and not all feasible (or optimal) solutions of programs (2) or (9) remain feasible in (10). To avoid these drawbacks, we consider weights $(u, v) \geq 0$ feasible in program (10) if the following two conditions are true. First, we require that $X_{o}^{\top} v>0$. Second, constraints (10.2) should be satisfied only by DMUs $(X, Y) \in \mathcal{T}_{\mathrm{CRS}-\mathrm{TO}}$ such that $X^{\top} v>0$. (As noted, this includes DMU $\left(X_{o}, Y_{o}\right)$.) Stating these two implicit conditions in program (10) is straightforward but is not implemented to preserve similarity with the standard linear fractional model of Charnes et al. (1978).

${ }^{8}$ Similar to the treatment of program $(10)$, we need several additional implicit conditions that define the feasible region of program (11). These are formally stated in the proof of Theorem 3.
} 


$$
\begin{aligned}
\theta_{3}^{*} & =\max _{u, v \geq 0}\left(\frac{Y_{o}^{\top} u / X_{o}^{\top} v}{\sup _{(X, Y) \in \mathcal{T}_{\mathrm{CRS}-\mathrm{TO}}}\left\{Y^{\top} u / X^{\top} v\right\}}\right) \\
& =\max _{u, v \geq 0}\left(\inf _{(X, Y) \in \mathcal{T}_{\mathrm{CRS}-\mathrm{TO}}}\left\{\frac{Y_{o}^{\top} u / X_{o}^{\top} v}{Y^{\top} u / X^{\top} v}\right\}\right) .
\end{aligned}
$$

Theorem 3. If solution $\left(u^{*}, v^{*}\right)$ is optimal in program (2), then $\left(u^{*}, v^{*}\right)$ is optimal in program (11). Therefore, the maximum in program (11) is attained and $\theta^{*}=\theta_{3}^{*}$.

According to the above result, the weights $\left(u^{*}, v^{*}\right)$ maximize the efficiency ratio of $\mathrm{DMU}_{o}$ in comparison to all DMUs in technology $\mathcal{T}_{\mathrm{CRS}-\mathrm{TO}}$, when no upper bound on such ratios is specified. This means that the upper bound of 1 on the efficiency ratios in inequalities $(10.2)$ is unimportant, and the weights $\left(u^{*}, v^{*}\right)$ are the most favorable to $\mathrm{DMU}_{o}$ among all nonnegative weights, without any additional conditions.

\section{The case of VRS}

Similar interpretation of optimal weights is obtained in the case of VRS. In order to avoid repetition, this is only briefly outlined below. The VRS analogue of the input-oriented multiplier model $(2)$ is stated with the additional free variable $u_{0}$ :

$$
\begin{aligned}
\hat{\theta}^{*}=\max & Y_{o}^{\top} u+u_{0} \\
\text { subject to } & X_{o}^{\top} v=1, \\
& Y_{j}^{\top} u-X_{j}^{\top} v+u_{0} \leq 0, \quad j=1, \ldots, N, \\
& Q_{t}^{\top} u-P_{t}^{\top} v \leq 0, \quad t=1, \ldots, K, \\
& u, v \geq 0, u_{0} \text { sign free. }
\end{aligned}
$$

The corresponding dual envelopment model is program (3), with the additional normalizing equality

$$
\sum_{j=1}^{N} \lambda_{j}=1
$$

Definition 2 (Podinovski 2004b). Technology $\mathcal{T}_{\text {VRS-TO }}$ is the set of all pairs $(X, Y) \in$ $\mathbb{R}_{+}^{m+s}$ for which there exist vectors $\lambda, \pi, S_{X}, S_{Y} \geq 0$ of appropriate dimensions such that equalities (5) and the normalizing equality (13) are true.

Consider the program with the infinite number of linear constraints:

$$
\begin{aligned}
\hat{\theta}_{1}^{*}=\max & Y_{o}^{\top} u+u_{0} \\
\text { subject to } & X_{o}^{\top} v=1 \\
& Y^{\top} u-X^{\top} v+u_{0} \leq 0, \quad \forall(X, Y) \in \mathcal{T}_{\mathrm{VRS}-\mathrm{TO}}, \\
& u, v \geq 0, u_{0} \text { sign free. }
\end{aligned}
$$

Denote $\hat{\Omega}$ and $\hat{\Omega}^{*}$ the sets of feasible and, respectively, optimal solutions to program (12). Similarly, let $\hat{\Omega}_{1}$ and $\hat{\Omega}_{1}^{*}$ be, respectively, the feasible and optimal sets of program (14). 
Theorem 4. If solution $\left(u, v, u_{0}\right)$ is feasible in program (12), then $\left(u, v, u_{0}\right)$ is feasible in (14). If $\left(u^{*}, v^{*}, u_{0}^{*}\right)$ is optimal in (12), then $\left(u^{*}, v^{*}, u_{0}^{*}\right)$ is optimal in (14). Therefore, the maximum in program (14) is attained, $\hat{\Omega} \subseteq \hat{\Omega}_{1}, \hat{\Omega}^{*} \subseteq \hat{\Omega}_{1}^{*}$ and $\hat{\theta}^{*}=\hat{\theta}_{1}^{*}$.

As in the case of CRS, program (14) can be restated in the linear fractional form:

$$
\begin{aligned}
\hat{\theta}_{2}^{*}=\max & \left(Y_{o}^{\top} u+u_{0}\right) / X_{o}^{\top} v \\
\text { subject to } & \left(Y^{\top} u+u_{0}\right) / X^{\top} v \leq 1, \quad \forall(X, Y) \in \mathcal{T}_{\mathrm{VRS}-\mathrm{TO}}, \\
& u, v \geq 0, u_{0} \text { sign free. }
\end{aligned}
$$

Theorem 5. If solution $\left(u^{*}, v^{*}, u_{0}^{*}\right)$ is optimal in program (12), then $\left(u^{*}, v^{*}, u_{0}^{*}\right)$ is optimal in program (15). Therefore, the maximum in program (15) is attained and $\hat{\theta}^{*}=\hat{\theta}_{2}^{*}$.

The proof of Theorem 5 is a straightforward adaptation of the proof of Theorem 2 and is not given. Furthermore, as in the case of CRS, program (15) can be restated in the maximin form similar to (11). An analogue of Theorem 3 for this case is also straightforward.

As follows from the above development, the interpretation of the optimal weights $\left(u^{*}, v^{*}, u_{0}^{*}\right)$ to the VRS multiplier model (12) is similar to the case of CRS. Namely, such weights are the most favorable for $\mathrm{DMU}_{o}$ when it is compared to all DMUs in the expanded technology $\mathcal{T}_{\text {VRS-TO }}$

\section{Summary and discussion}

To be specific, we limit our discussion to the optimal solutions of the input-oriented multiplier models under the assumption of CRS. The same observations extend, with obvious modifications, to the output-oriented CRS models and the models stated under the assumption of VRS.

The conventional interpretation given by Charnes et al. (1978) to any optimal weights $\left(u^{*}, v^{*}\right)$ to the standard (without weight restrictions) multiplier CRS model states that such weights represent $\mathrm{DMU}_{o}$ in the best light in comparison to all observed DMUs. As proved by Podinovski (2001a), the same interpretation remains valid for multiplier models with weight restrictions, provided the latter are all homogeneous and not linked. For ease of reference, we state this as follows:

Interpretation 1. Let all (homogeneous) weight restrictions (2.4) be not linked. Then any optimal weights $\left(u^{*}, v^{*}\right)$ to the multiplier model (2) are the most favorable for $\mathrm{DMU}_{o}$ if the latter is benchmarked against all observed DMUs.

Interpretation 1 means that, for the weights $\left(u^{*}, v^{*}\right)$, the efficiency ratio $Y_{o}^{\top} u / X_{o}^{\top} v$ of $\mathrm{DMU}_{o}$ attains its maximum in relation to the maximum of similar ratios across all observed DMUs. As shown by examples in Podinovski and Athanassopoulos (1998) and later literature, Interpretation 1 is no longer valid in the general case that includes linked or nonhomogeneous weight restrictions. This implies that the meaning of efficiency of $\mathrm{DMU}_{o}$ in terms of the multiplier model with arbitrary weight restrictions has so far remained unclear.

In contrast, the interpretation of efficiency in terms of the envelopment model is far more complete. Podinovski (2004b) shows that the incorporation of weight restrictions of any types in the multiplier model leads to the expansion of the model of technology by means of dual terms (trade-offs). The envelopment model projects $\mathrm{DMU}_{o}$ on the boundary of this technology (in input or output orientation). In other words, this model benchmarks $\mathrm{DMU}_{o}$ against all DMUs in the entire expanded technology, and not only against the observed DMUs. 
The above observations indicate two problems with the conventional interpretation.

First, while the efficiency of $\mathrm{DMU}_{o}$ can be conventionally interpreted as the proportional improvement factor in the envelopment model with any weight restrictions (taking on the dual form of production trade-offs), the multiplier model allows the conventional interpretation of efficiency only for some types of weight restriction.

Second, even for homogeneous and not linked weight restrictions, for which both models provide a meaningful interpretation, the two models benchmark $\mathrm{DMU}_{o}$ against different sets of DMUs. As noted, for the multiplier model with weight restrictions this includes the set of observed DMUs only, and for the dual envelopment model this includes the set of all DMUs in the technology, including unobserved units.

Our development overcomes the above discrepancies by changing the approach to the interpretation of the multiplier model. Based on Theorems 1-3 and their discussion, we summarize our main results as follows.

Interpretation 2. Any optimal weights $\left(u^{*}, v^{*}\right)$ to the multiplier model (2) with weight restrictions (2.4) (and, as shown in Remark 1, with any weight restrictions (1)) are the most favorable for $\mathrm{DMU}_{o}$ if the latter is benchmarked against all DMUs in the entire technology $\mathcal{T}_{\text {CRS-TO }}$. Furthermore, the weights $\left(u^{*}, v^{*}\right)$, while satisfying the weight restrictions because of the constraints of model (2), are the best for $\mathrm{DMU}_{o}$ among all nonnegative weights $(u, v)$, and not only among those that satisfy the weight restrictions.

If all weight restrictions are homogeneous and not linked, then both Interpretations 1 and 2 are applicable. Otherwise, only the latter is correct. For example, both interpretations apply to the standard multiplier CRS model without weight restrictions. Namely, any optimal weights $\left(u^{*}, v^{*}\right)$ in this model show $\mathrm{DMU}_{o}$ in the best light in comparison to the set of observed DMUs and also in comparison to all DMUs in the standard CRS technology. 


\section{Appendix A. Proofs}

Lemma 1. The optimal value $\theta_{1}^{*}$ of program (9) is attained, and $\theta^{*}=\theta_{1}^{*}$.

Proof of Lemma 1. $\mathcal{T}_{\mathrm{CRS}-\mathrm{TO}}$ is a polyhedral cone (Podinovski 2015). By Theorem 19.1 in Rockafellar (1970), it is generated by a finite set of directions $\left(A_{k}, B_{k}\right) \in \mathbb{R}^{m+s}, k=1, \ldots, k^{\prime}$. Therefore $\mathcal{T}_{\mathrm{CRS}-\mathrm{TO}}$ is the set of all pairs $(X, Y)$ for which there exists a vector $\mu \in \mathbb{R}_{+}^{k^{\prime}}$ such that

$$
X=\sum_{k=1}^{k^{\prime}} \mu_{k} A_{k}, Y=\sum_{k=1}^{k^{\prime}} \mu_{k} B_{k} .
$$

Then program (3) is restated as follows:

$$
\begin{array}{ll}
\theta^{*}=\min & \theta \\
\text { subject to } & \sum_{k=1}^{k^{\prime}} \mu_{k} A_{k}=\theta X_{o}, \\
& \sum_{k=1}^{k^{\prime}} \mu_{k} B_{k}=Y_{o}, \\
& \mu \geq 0, \theta \text { sign free. }
\end{array}
$$

By Definition 1, $\mathcal{T}_{\mathrm{CRS}-\mathrm{TO}}$ satisfies free disposability, and we can replace equalities in (A.2.2) and (A.2.3) by the appropriate inequalities. Taking the dual, we have:

$$
\begin{aligned}
\theta^{*}=\max & Y_{o}^{\top} u \\
\text { subject to } & X_{o}^{\top} v=1, \\
& B_{k}^{\top} u-A_{k}^{\top} v \leq 0, \quad k=1, \ldots, k^{\prime}, \\
& u, v \geq 0 .
\end{aligned}
$$

Any $(X, Y) \in \mathcal{T}_{\mathrm{CRS}-\mathrm{TO}}$ satisfies (A.1) with some vector $\mu \geq 0$. Multiplying each inequality $k=1, \ldots, k^{\prime}$ in (A.3.3) by $\mu_{k} \geq 0$, and adding the resulting inequalities, we obtain

$$
Y^{\top} u-X^{\top} v \leq 0, \quad \forall(X, Y) \in \mathcal{T}_{\mathrm{CRS}-\mathrm{TO}}
$$

Because (A.3.3) implies (A.4), the incorporation of (A.4) in (A.3) does not affect its feasible region. By (A.1), (A.4) implies (A.3.3). Omitting all inequalities (A.3.3) as redundant but keeping (A.4), we obtain (2). Therefore, $\theta_{1}^{*}=\theta^{*}$ and is attained at any optimal solution to (A.3).

Proof of Theorem 1. Let $(u, v) \in \Omega$. We need to prove that $(u, v) \in \Omega_{1}$. It suffices to show that $(u, v)$ satisfies $(9.3), \forall(X, Y) \in \mathcal{T}_{\text {CRS-TO }}$. Indeed, any such unit $(X, Y)$ satisfies $(5)$ with some vectors $\lambda, \pi, S_{X}, S_{Y} \geq 0$. Multiplying each inequality in (2.2) and (2.3) by the corresponding components $\lambda_{j}$ and $\pi_{t}$, adding the resulting inequalities and noting that $S_{X}, S_{Y} \geq 0$, we obtain (9.3), as required. Finally, let $\left(u^{*}, v^{*}\right) \in \Omega^{*}$. Then, as proved, $\left(u^{*}, v^{*}\right)$ is feasible in (9). From program (2), $Y_{o}^{\top} u^{*}=\theta^{*}$. By Lemma $1, \theta^{*}=\theta_{1}^{*}$. Therefore, $\left(u^{*}, v^{*}\right) \in \Omega_{1}^{*}$.

Lemma 2. The feasible region of program (9) is unchanged if constraints (9.3) are required only for DMUs $(X, Y)$ for which $X^{\top} v>0$, i.e., if inequalities (9.3) are replaced by

$$
Y^{\top} u-X^{\top} v \leq 0, \quad \forall(X, Y) \in \mathcal{T}_{\mathrm{CRS}-\mathrm{TO}}: X^{\top} v>0 .
$$


Proof of Lemma 2. We need to prove that, if $(u, v)$ satisfies (9.2), (9.4) and (A.5), then

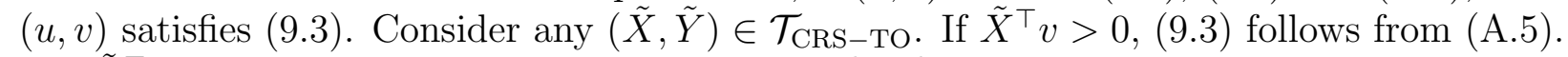
Let $\tilde{X}^{\top} v=0$. Consider the sequence of units $\left(X^{k}, Y^{k}\right), k=1,2, \ldots$, defined as follows:

$$
\left(X^{k}, Y^{k}\right)=(\tilde{X}, \tilde{Y})+(1 / k)\left(X_{o}, Y_{o}\right) .
$$

Because $\mathcal{T}_{\text {CRS-TO }}$ is a polyhedral cone (Podinovski 2015), $\left(X^{k}, Y^{k}\right) \in \mathcal{T}_{\text {CRS-TO }}, \forall k$. From $(9.2)$ and (A.6), $\left(X^{k}\right)^{\top} v>0, \forall k$. By (A.5), we have $\left(Y^{k}\right)^{\top} u-\left(X^{k}\right)^{\top} v \leq 0, \forall k$. Taking $k \rightarrow+\infty$, we have $\tilde{Y}^{\top} u-\tilde{X}^{\top} v \leq 0$, and (9.3) follows.

Proof of Proposition 1. Define $(\tilde{X}, \tilde{Y})=\left(\theta_{1}^{*} X_{o}, Y_{o}\right)=\left(\theta^{*} X_{o}, Y_{o}\right)$. By Remark 1, $\theta^{*}>0$.

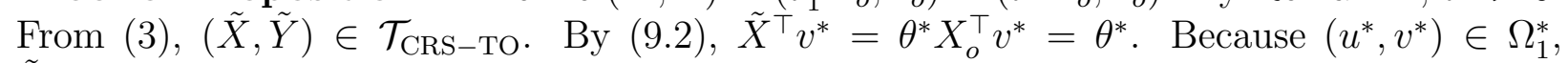
$\tilde{Y}^{\top} u^{*}=\theta^{*}$. Therefore,

$$
\tilde{Y}^{\top} u^{*}-\tilde{X}^{\top} v^{*}=0
$$

Proof of Proposition 2. By Theorem 1, it suffices to prove that $\Omega_{1}^{*} \subseteq \Omega^{*}$. Let $\left(u^{*}, v^{*}\right) \in$ $\Omega_{1}^{*}$. Because the objective functions in (2) and (9) are the same, it suffices to prove that $\left(u^{*}, v^{*}\right) \in \Omega$. Because $\left(X_{j}, Y_{j}\right) \in \mathcal{T}_{\mathrm{CRS}-\mathrm{TO}}, \forall j=1, \ldots, N,(9.3)$ implies $(2.3)$, and the latter is satisfied by $\left(u^{*}, v^{*}\right)$. It remains to prove that $\left(u^{*}, v^{*}\right)$ satisfies weight restrictions $(2.4)$.

Assume there exists a $t^{\prime}$ for which the inequality (2.4) is not true, i.e., we have

$$
Q_{t^{\prime}}^{\top} u^{*}-P_{t^{\prime}}^{\top} v^{*}>0
$$

Consider the unit $(\tilde{X}, \tilde{Y})>0$ defined in the proof of Proposition 1. Because $(\tilde{X}, \tilde{Y})>0$, there exists a small $\pi_{t^{\prime}}>0$ such that $(\hat{X}, \hat{Y})=\left(\tilde{X}+\pi_{t^{\prime}} P_{t^{\prime}}, \tilde{Y}+\pi_{t^{\prime}} Q_{t^{\prime}}\right) \geq 0$. Therefore, $(\hat{X}, \hat{Y}) \in \mathcal{T}_{\mathrm{CRS}-\mathrm{TO}}$. From (A.7) and (A.8), we have $\hat{Y}^{\top} u^{*}-\hat{X}^{\top} v^{*}>0$, which contra$\operatorname{dicts}(9.3)$.

Proof of Theorem 2. By Theorem $1,\left(u^{*}, v^{*}\right)$ is optimal in (9) and $\theta^{*}=\theta_{1}^{*}$. By footnote 3 , $\left(u^{*}, v^{*}\right)$ is feasible in (10). Assume that $\left(u^{*}, v^{*}\right)$ is not optimal in (10). Then there exists a feasible solution $\left(u^{\prime}, v^{\prime}\right)$ to $(10)$ such that $Y_{o}^{\top} u^{\prime} / X_{o}^{\top} v^{\prime}=\theta^{\prime}$ and $\theta^{\prime}>\theta_{1}^{*}$. Then for any $\alpha>0,\left(\alpha u^{\prime}, \alpha v^{\prime}\right)$ is also feasible in program (10), and the corresponding objective function $\left(Y_{o}^{\top} \alpha u^{\prime}\right) /\left(X_{o}^{\top} \alpha v^{\prime}\right)=\theta^{\prime}$. Define $\alpha^{\prime}$ such that $X_{o}^{\top} \alpha^{\prime} v^{\prime}=1$. (By footnote $3, X_{o}^{\top} v^{\prime}>0$, and we have $\alpha^{\prime}=1 /\left(X_{o}^{\top} v^{\prime}\right)$.) Then $\left(\alpha^{\prime} u^{\prime}, \alpha^{\prime} v^{\prime}\right)$ is feasible in (9). Indeed, inequalities (10.2) are true for all $(X, Y) \in \mathcal{T}_{\mathrm{CRS}-\mathrm{TO}}$ such that $X^{\top} v>0$. This implies (A.5). By Lemma 2, this implies (9.3). The corresponding value of the objective function (9.1) is equal to $\theta^{\prime}>\theta_{1}^{*}$, which contradicts the optimality of $\theta_{1}^{*}$ in (9).

Proof of Proposition 3. Define $\alpha=1 /\left(X_{o} v^{*}\right)>0$. Then $\left(u^{\prime}, v^{\prime}\right)=\left(\alpha u^{*}, \alpha v^{*}\right)$ is also optimal in (10). Repeating the end of the proof of Theorem 2, $\left(u^{\prime}, v^{\prime}\right)$ is feasible in (9). Because $\theta_{2}^{*}=\left(Y_{o}^{\top} u^{\prime}\right) /\left(X_{o}^{\top} v^{\prime}\right)=Y_{o}^{\top} u^{\prime}$ and $\theta_{2}^{*}=\theta_{1}^{*},\left(u^{\prime}, v^{\prime}\right)$ is optimal in (9). By Proposition 1 , $Y_{o}^{\top} u^{\prime}=\theta^{*} X_{o}^{\top} v^{\prime}$. Dividing this equality by $\alpha$, we obtain $Y_{o}^{\top} u^{*}=\theta^{*} X_{o}^{\top} v^{*}$, and the proof follows.

Proof of Theorem 3. To address potential division by zero in program (11), we clarify its statement as follows:

$$
\theta_{3}^{*}=\max _{(u, v) \in \Omega_{3}}\left(\frac{Y_{o}^{\top} u / X_{o}^{\top} v}{\sup _{(X, Y) \in \mathcal{T}_{u, v}}\left\{Y^{\top} u / X^{\top} v\right\}}\right)
$$


where the sets $\Omega_{3}$ and $\mathcal{T}_{u, v}$ are defined as follows:

$$
\begin{aligned}
& \Omega_{3}=\left\{(u, v) \geq 0 \mid X_{o}^{\top} v>0, Y_{o}^{\top} u>0\right\}, \\
& \mathcal{T}_{u, v}=\left\{(X, Y) \in \mathcal{T}_{\mathrm{CRS}-\mathrm{TO}} \mid X^{\top} v>0\right\} .
\end{aligned}
$$

Note that $\left(u^{*}, v^{*}\right) \in \Omega_{3}$ and hence $\Omega_{3} \neq \varnothing$. Indeed, by (2.2), $X_{o}^{\top} v^{*}=1>0$ and, by (2.1) and Remark $2, Y_{o}^{\top} u^{*}=\theta^{*}>0$. Furthermore, for any $(u, v) \in \Omega_{3},\left(X_{o}, Y_{o}\right) \in \mathcal{T}_{u, v}$. Therefore, for all $(u, v) \in \Omega_{3}$, the set $\mathcal{T}_{u, v} \neq \varnothing$ and the supremum in the denominator of (A.9) is greater than zero. If for some weights $(u, v) \in \Omega_{3}$ the supremum in the denominator of (A.9) is unbounded, the expression in parentheses is considered to be equal to zero - see Example 3 in Appendix B.

By Proposition 3, for the weights $\left(u^{*}, v^{*}\right)$, the objective function (expression in parentheses) of program (A.9) is equal to $\theta^{*}$. Therefore we have $\theta_{3}^{*} \geq \theta^{*}$.

Suppose $\left(u^{*}, v^{*}\right)$ is not optimal in program (A.9). Then there exists a $\left(u^{\prime}, v^{\prime}\right) \in \Omega_{3}$ such that

$$
\psi\left(u^{\prime}, v^{\prime}\right)=\frac{Y_{o}^{\top} u^{\prime} / X_{o}^{\top} v^{\prime}}{\sup _{(X, Y) \in \mathcal{T}_{u^{\prime}, v^{\prime}}}\left\{Y^{\top} u^{\prime} / X^{\top} v^{\prime}\right\}}=\theta^{\prime}>\theta^{*} .
$$

Note that, for any $\alpha>0,\left(u^{\prime}, \alpha v^{\prime}\right) \in \Omega_{3}$ and $\psi\left(u^{\prime}, \alpha v^{\prime}\right)=\psi\left(u^{\prime}, v^{\prime}\right)=\theta^{\prime}$. Define

$$
\alpha^{\prime}=\sup _{(X, Y) \in \mathcal{T}_{u^{\prime}, v^{\prime}}}\left\{Y^{\top} u^{\prime} / X^{\top} v^{\prime}\right\}
$$

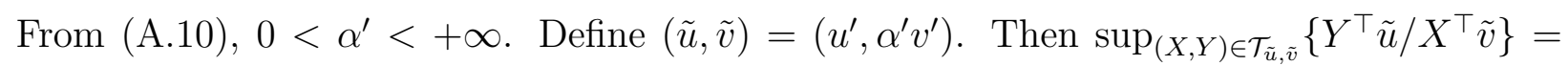
1 and $\psi(\tilde{u}, \tilde{v})=Y_{o}^{\top} \tilde{u} / X_{o}^{\top} \tilde{v}=\theta^{\prime}$. Therefore, $(\tilde{u}, \tilde{v})$ is feasible in program (10), and the corresponding value of the objective function (10.1) is equal to $\theta^{\prime}$. By (A.10), $\theta^{\prime}>\theta^{*}$. This contradicts Theorem 2 , according to which $\theta^{*}$ is the optimal value of program (10).

Lemma 3. The maximum in program (14) is attained and $\hat{\theta}^{*}=\hat{\theta}_{1}^{*}$.

Proof of Lemma 3. The proof is similar to the proof of Lemma 1. Because $\mathcal{T}_{\text {VRS-TO }}$ is a polyhedral set (Podinovski 2015), it is generated by a finite number of points $\left(A_{k}, B_{k}\right) \in$ $\mathbb{R}^{m+s}, k=1, \ldots, \tilde{k}$, and directions $\left(A_{k}, B_{k}\right) \in \mathbb{R}^{m+s}, k=\tilde{k}+1, \ldots, k^{\prime}$ (Rockafellar 1970, Theorem 19.1). Therefore $\mathcal{T}_{\text {VRS-TO }}$ is the set of all units $(X, Y)$ for which there exists a vector $\mu \in \mathbb{R}_{+}^{k^{\prime}}$ such that

$$
X=\sum_{k=1}^{k^{\prime}} \mu_{k} A_{k}, \quad Y=\sum_{k=1}^{k^{\prime}} \mu_{k} B_{k}, \quad \sum_{k=1}^{\tilde{k}} \mu_{k}=1 .
$$

Repeating the proof of Lemma 1, we have

$$
\begin{aligned}
\hat{\theta}^{*}=\max & Y_{o}^{\top} u+u_{o} \\
\text { subject to } & X_{o}^{\top} v=1, \\
& B_{k}^{\top} u-A_{k}^{\top} v+u_{o} \leq 0, \quad k=1, \ldots, \tilde{k}, \\
& B_{k}^{\top} u-A_{k}^{\top} v \leq 0, \quad k=\tilde{k}+1, \ldots, k^{\prime}, \\
& u, v \geq 0 .
\end{aligned}
$$

Any $(X, Y) \in \mathcal{T}_{\text {VRS-TO }}$ satisfies (A.11) with some $\mu \geq 0$. Multiplying each inequality $k=1, \ldots, k^{\prime}$ in (A.12.3) and (A.12.4) by $\mu_{k} \geq 0$ and adding the resulting inequalities, we obtain

$$
Y^{\top} u-X^{\top} v+u_{0} \leq 0, \quad \forall(X, Y) \in \mathcal{T}_{\mathrm{VRS}-\mathrm{TO}}
$$


Because inequalities (A.12.3) and (A.12.4) imply (A.13), the incorporation of inequalities (A.13) in program (A.12) does not affect its feasible region. Note that in the resulting program, constraints (A.12.3) follow from (A.13) and can be omitted. Let us prove that inequalities (A.12.4) also follow from (A.13). Consider any $k=\tilde{k}+1, \ldots, k^{\prime}$. By (A.11), $(X, Y)=\left(A_{1}+\mu_{k} A_{k}, B_{1}+\mu_{k} B_{k}\right) \in \mathcal{T}_{\mathrm{VRS}-\mathrm{TO}}, \forall \mu_{k}>0$. The corresponding inequality (A.13) takes on the form:

$$
\left(B_{1}+\mu_{k} B_{k}\right)^{\top} u-\left(A_{1}+\mu_{k} A_{k}\right)^{\top} v+u_{0} \leq 0 .
$$

Let $\left(u, v, u_{o}\right)$ satisfy (A.13) and, therefore, (A.14). Dividing both sides of (A.14) by $\mu_{k}>0$ and taking $\mu_{k} \rightarrow+\infty$, we obtain inequality $k$ in (A.12.4). Therefore, all inequalities (A.12.4) follow from (A.13) and can be omitted. Consequently, program (A.12) can be restated as $(14)$, and $\hat{\theta}^{*}=\hat{\theta}_{1}^{*}$.

Proof of Theorem 4. The proof is similar to the proof of Theorem 1. Let $\left(u, v, u_{0}\right) \in \hat{\Omega}$. We need to prove that $\left(u, v, u_{0}\right)$ satisfies $(14.3), \forall(X, Y) \in \mathcal{T}_{\text {VRS-TO. Indeed, }(X, Y) \text { satisfies }}$ Definition 2 with some vectors $\lambda, \mu, S_{X}, S_{Y} \geq 0$. Multiplying (12.2) and (12.3) by the corresponding $\lambda_{j}$ and $\pi_{t}$, adding the resulting inequalities and noting that $S_{X}, S_{Y} \geq 0$, we obtain (14.3). Therefore, $\left(u, v, u_{0}\right) \in \hat{\Omega}_{1}$. Finally, let $\left(u^{*}, v^{*}, u_{0}^{*}\right) \in \hat{\Omega}^{*}$. Then, as proved, $\left(u^{*}, v^{*}, u_{0}^{*}\right) \in \hat{\Omega}_{1}$. From (12), $Y_{o}^{\top} u^{*}+u_{0}^{*}=\hat{\theta}^{*}$. By Lemma 3, $\hat{\theta}^{*}=\hat{\theta}_{1}^{*}$. Therefore, $\left(u^{*}, v^{*}, u_{0}^{*}\right) \in$ $\hat{\Omega}_{1}$. 


\section{Appendix B. Further examples}

Example 2. This example is complementary to Section 4.1. It shows that generally $\Omega \neq \Omega_{1}$ and $\Omega^{*} \neq \Omega_{1}^{*}$, and also illustrates Proposition 2. Consider technology $\mathcal{T}_{\mathrm{CRS}-\mathrm{TO}}$ generated by the single DMU $A$ shown in Table A.3 and the single weight restriction $u_{1}-u_{2} \leq 0$. This corresponds to the following production trade-off that affects only outputs: $P=(0)$, $Q=(1,-1)^{\top}$.

Table A.3: DMUs in Example 2.

\begin{tabular}{llll}
\hline DMU & Input & Output 1 & Output 2 \\
\hline$A$ & 1 & 1 & 1 \\
$B$ & 1 & 2 & 0 \\
\hline
\end{tabular}

Figure A.2 shows the section of technology $\mathcal{T}_{\mathrm{CRS}-\mathrm{TO}}$ in the output dimensions, for the level of input equal to 1 . In particular, the unit square $O D A C$ is the section of the standard CRS technology (without the weight restriction) generated by DMU $A$.

According to formula (5), the trade-off $(P, Q)$ expands the technology as follows. Applying trade-off $Q=(1,-1)^{\top}$ to DMU $A$ in proportion $\pi=1$, we add 1 to Output 1 and simultaneously subtract 1 from Output 2. This adds DMU $B$ to the technology. Applying the same trade-off in proportions $0<\pi<1$, we generate the line $A B$. By the assumption of free disposability, the entire triangle $A B C$ is added to the technology. Overall, the section of technology $\mathcal{T}_{\mathrm{CRS}-\mathrm{TO}}$ in the output dimensions is polyhedron $A B O D$. (The full technology is the polyhedral cone generated by this section.)

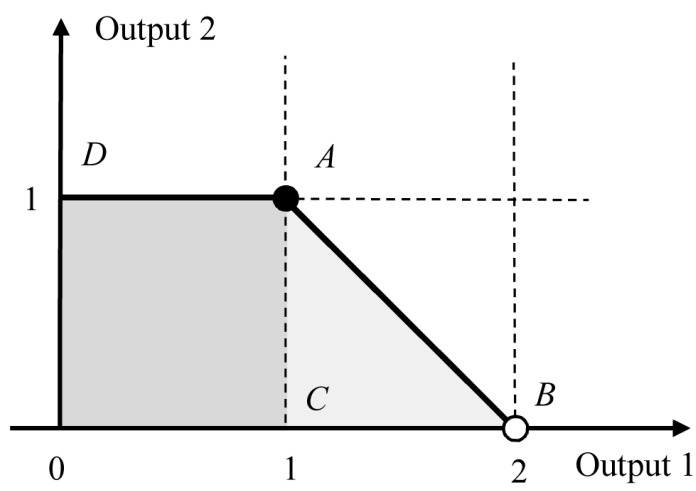

Figure A.2: Technology $\mathcal{T}_{\mathrm{CRS}-\mathrm{TO}}$ in Example 2.

Consider programs (2) and (9). The former is stated as follows (we note that $v_{1}=1$ ):

$$
\begin{aligned}
\theta^{*}=\max & 1 u_{1}+1 u_{2} \\
\text { subject to } & 1 v_{1}=1 \\
& 1 u_{1}+1 u_{2} \leq 1 \\
& 1 u_{1}-1 u_{2} \leq 0 \\
& u_{1}, u_{2}, v_{1} \geq 0
\end{aligned}
$$




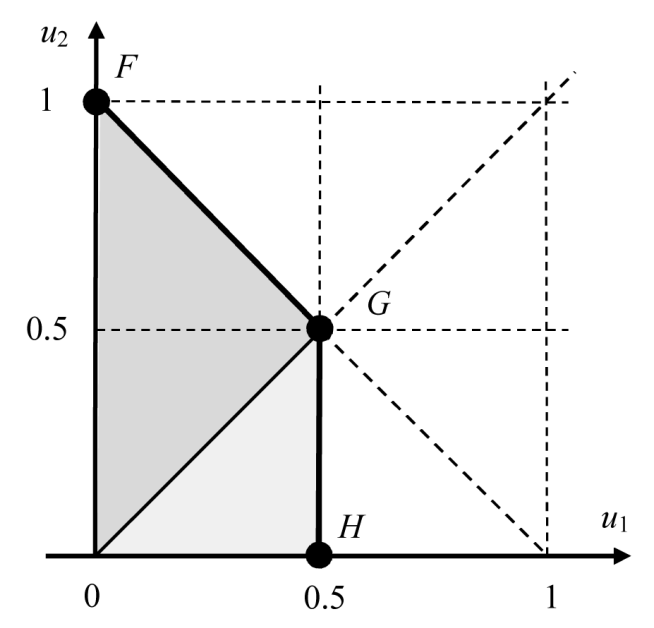

Figure A.3: Feasible regions and optimal solutions in Example 2.

The feasible region $\Omega$ of the above program consists of all vectors $\left(u_{1}, u_{2}, v_{1}\right)$ such that $v_{1}=1$ and the subvector $\left(u_{1}, u_{2}\right)$ is any point in the shaded triangle $O F G$ in Figure A.3.

Now refer to program (9). From Figure A.2 observe that $\mathcal{T}_{\mathrm{CRS}-\mathrm{TO}}$ coincides with the standard CRS technology generated by DMUs $A$ and $B$ (without weight restrictions). It is straightforward to verify that the feasible region of program (9) remains unchanged if the infinite number of inequalities (9.3) are replaced by just two inequalities for DMUs $A$ and $B$. This leads to the following program:

$$
\begin{aligned}
\theta_{1}^{*}=\max & 1 u_{1}+1 u_{2} \\
\text { subject to } & 2 u_{1}-0 u_{2} \leq 0 \\
\text { and } & (\mathrm{A} .15 .2),(\mathrm{A} .15 .3) \text { and }(\mathrm{A} .15 .5)
\end{aligned}
$$

The feasible region $\Omega_{1}$ of this program (in terms of output weights $u_{1}$ and $u_{2}$ ) is the shaded polyhedron $O F G H$ in Figure A.3. Observe that $\Omega \neq \Omega_{1}$.

Furthermore, the sets of optimal solutions $\Omega^{*}$ and $\Omega_{1}^{*}$ to programs (A.15) and (A.16) (in terms of weights $u_{1}$ and $u_{2}$ ) are identical and coincide with the line segment $F G$. This observation is consistent with Proposition 2 -note that all inputs and outputs of DMU $A$ are strictly positive.

Now consider the assessment of efficiency of DMU $B$ whose Output 2 is zero. ${ }^{9}$ This requires changing the objective functions of programs (A.15) and (A.16) to

$$
2 u_{1}+0 u_{2} .
$$

In program (A.15) with its objective function replaced by (A.17), the only optimal weights $\left(u_{1}^{*}, u_{2}^{*}\right)=(0.5,0.5)$ correspond to the single point $G$. In the similarly modified program (A.16), the entire segment $G H$ represents optimal weights $\left(u_{1}^{*}, u_{2}^{*}\right)$. This shows that, if the assumption of Proposition 2 is not true (note that DMU $B$ does not satisfy it), then generally $\Omega^{*} \neq \Omega_{1}^{*}$.

Example 3. This example shows that the supremum in the denominator of program (11) may be unbounded (equal to $+\infty$ ), as noted in the proof of Theorem 3. Consider technology

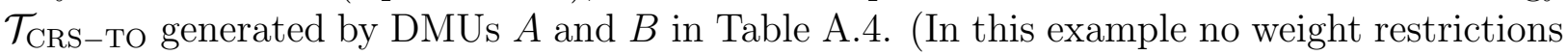
are specified, and $\mathcal{T}_{\mathrm{CRS}-\mathrm{TO}}$ is the standard CRS technology.)

\footnotetext{
${ }^{9}$ The fact that DMU $B$ is unobserved is unimportant: we can always expand the original data set and include $B$ as an observed DMU. This does not change our example.
} 
Table A.4: DMUs in Example 3.

\begin{tabular}{llll}
\hline DMU & Input 1 & Input 2 & Output \\
\hline$A$ & 1 & 0 & 1 \\
$B$ & 0 & 1 & 1 \\
$C(\lambda)$ & $\lambda$ & $1-\lambda$ & 1 \\
\hline
\end{tabular}

Define DMU $C(\lambda)=\lambda A+(1-\lambda) B \in \mathcal{T}_{\mathrm{CRS}-\mathrm{TO}}$, for all $\lambda \in[0,1]$. The input and output vectors of DMU $C(\lambda)$ are, respectively, $X(\lambda)=(\lambda, 1-\lambda)^{\top}$ and $Y(\lambda)=(1)$, as shown in Table A.4.

Suppose $\left(X_{o}, Y_{o}\right)$ in program (11) is DMU $A$. Consider the weights $\left(u_{1}^{\prime}, v_{1}^{\prime}, v_{2}^{\prime}\right)=(1,1,0)$. Then $X_{0}^{\top} v^{\prime}=1$ and $Y_{0}^{\top} u^{\prime}=1$.

Note that $X(\lambda) v^{\prime}=\lambda v_{1}^{\prime}+(1-\lambda) v_{2}^{\prime}=\lambda>0$ and $Y(\lambda) u^{\prime}=1 u_{1}^{\prime}=1$. The ratio $\left(Y(\lambda) u^{\prime}\right) /\left(X(\lambda) v^{\prime}\right) \rightarrow+\infty$ as $\lambda \rightarrow 0+$. This shows that, for the weights $\left(u_{1}^{\prime}, v_{1}^{\prime}, v_{2}^{\prime}\right)$, the supremum in (11) is unbounded, even if taken with respect to DMUs $(X, Y) \in \mathcal{T}_{\mathrm{CRS}-\mathrm{TO}}$ for which $X^{\top} v^{\prime}>0$. 


\section{References}

Allen, R., Athanassopoulos, A., Dyson, R.G., Thanassoulis, E. (1997). Weights restrictions and value judgements in data envelopment analysis: Evolution, development and future directions. Annals of Operations Research, 73, 13-34.

Banker, R.D., Charnes, A., Cooper, W.W. (1984). Some models for estimating technical and scale efficiencies in data envelopment analysis. Management Science, 30(9), 1078-1092.

Charnes, A., Cooper, W.W., Rhodes, E. (1978). Measuring the efficiency of decision making units. European Journal of Operational Research, 2(6), 429-444.

Charnes, A., Cooper, W.W., Wei, Q.L., Huang, Z.M. (1989). Cone ratio data envelopment analysis and multi-objective programming. International Journal of Systems Science, 20(7), 1099-1118.

Cook, W.D., Zhu, J. (2008). Context-dependent assurance regions in DEA. Operations Research, 56(1), 69-78.

Cooper, W.W., Seiford, L.M., Tone, K. (2007). Data envelopment analysis. A comprehensive text with models, applications, references and DEA-Solver software. (2nd Ed.) New York: Springer Science + Busines Media.

Dyson, R.G., Allen, R., Camanho, A.S., Podinovski, V.V., Sarrico, C., Shale, E.A. (2001). Pitfalls and protocols in DEA. European Journal of Operational Research, 132(2), 245-259.

Dyson, R.D., Thanassoulis, E. (1988). Reducing weight flexibility in data envelopment analysis. Journal of the Operational Research Society, 39(6), 563-576.

Halme, M., Korhonen, P. (2000). Restricting weights in value efficiency analysis. European Journal of Operational Research, 126(1), 175-188.

Jain, V., Kumar., A., Kumar, S., Chandra, C. (2015). Weight restrictions in data envelopment analysis: A comprehensive genetic algorithm based approach for incorporating value judgments. Expert Systems with Applications, 42(3), 1503-1512.

Joro, T., Korhonen, P.J. (2015). Extension of data envelopment analysis with preference information: Value efficiency. New York: Springer Science + Busines Media.

Khalili, M., Camanho, A.S., Portela, M.C.A.S., Alirezaee, M.R. (2010). The measurement of relative efficiency using data envelopment analysis with assurance regions that link inputs and outputs. European Journal of Operational Research, 203(3), 761-770.

Podinovski, V.V. (1999). Side effects of absolute weight bounds in DEA models. European Journal of Operational Research, 115(3), 583-595.

Podinovski, V.V. (2001a). DEA models for the explicit maximisation of relative efficiency. European Journal of Operational Research, 131(3), 572-586.

Podinovski, V.V. (2001b). Validating absolute weight bounds in data envelopment analysis (DEA) models. Journal of the Operational Research Society, 52(2), 221-225.

Podinovski, V.V. (2004a). Suitability and redundancy of non-homogeneous weight restrictions for measuring the relative efficiency in DEA. European Journal of Operational Research, 154(2), 380-395.

Podinovski, V.V. (2004b). Production trade-offs and weight restrictions in data envelopment analysis. Journal of the Operational Research Society, 55(12), 1311-1322.

Podinovski, V.V. (2005). The explicit role of weight bounds in models of data envelopment analysis. Journal of the Operational Research Society, 56(12), 1408-1418.

Podinovski, V.V. (2015). DEA models with production trade-offs and weight restrictions. In J. Zhu (Ed.), Data envelopment analysis: A handbook of models and methods (pp. 105-144). New York: Springer Science + Busines Media.

Podinovski, V.V., Athanassopoulos, A.D. (1998). Assessing the relative efficiency of decision making units using DEA models with weight restrictions. Journal of the Operational Research Society, 49(5), 500-508.

Podinovski, V.V., Bouzdine-Chameeva, T. (2013). Weight restrictions and free production in data envelopment analysis. Operations Research, 61(2), 426-437.

Podinovski, V.V., Bouzdine-Chameeva, T. (2015). Consistent weight restrictions in data envelopment analysis. European Journal of Operational Research, 244(1), 201-209.

Podinovski, V.V., Chambers, R.G., Atici, K.B., Deineko, I.D. (2016). Marginal values and returns to scale for nonparametric production frontiers. Operations Research, 64(1), 236-250.

Podinovski, V.V., Førsund, F.R. (2010). Differential characteristics of efficient frontiers in data envelopment analysis. Operations Research, 58(6), 1743-1754.

Podinovski, V.V., Førsund, F.R., Krivonozhko, V.E. (2009). A simple derivation of scale elasticity in data envelopment analysis. European Journal of Operational Research, 197(1), 149-153.

Rockafellar, R.T. (1970). Convex analysis. Princeton, NJ: Princeton University Press. 
Roll, Y., Cook, W.D., Golany, B. (1991). Controlling factor weights in data envelopment analysis. IIE Transactions, 23(1), 2-9.

Sahoo, B.K., Tone, K. (2015). Scale elasticity in non-parametric DEA approach. In J. Zhu (Ed.), Data envelopment analysis: A handbook of models and methods (pp. 269-290). New York: Springer Science + Busines Media.

Thanassoulis, E., Portela, M.C.S., Despić, O. (2008). Data envelopment analysis: The mathematical programming approach to efficiency analysis. In H.O. Fried, C.A.K. Lovell, S.S. Schmidt (Eds.), The measurement of productive efficiency and productivity growth (pp. 251-420). New York: Oxford University Press.

Thompson, R.G., Langemeier, L.N., Lee, C.T., Lee, E., Thrall, R.M. (1990). The role of multiplier bounds in efficiency analysis with application to Kansas farming. Journal of Econometrics, 46(1-2), 93-108.

Wong, Y.-H.B., Beasley, J.E. (1990). Restricting weight flexibility in data envelopment analysis. Journal of the Operational Research Society, 41(9), 829-835. 